\title{
Orbit Closures of Linear Algebraic Groups
}

\author{
Willem A. de Graaf \\ Department of Mathematics \\ University of Trento \\ Italy \\ degraaf@science.unitn.it
}

\begin{abstract}
Let an algebraic group be given, acting on a vector space. We consider the problem of deciding whether a given element of the vector space lies in the closure of the orbit of another given element. We describe three methods for dealing with this problem that have appeared in the literature. We illustrate the methods by examples.
\end{abstract}

\section{Introduction}

Actions of linear algebraic groups appear in many contexts. In some of them the following problem is of interest: given an algebraic group $G \subset \mathrm{GL}(V)$ and $v, w \in V$, decide whether $w$ lies in the (Zariski-) closure of the orbit $G \cdot v$. This problem arises for example in the theory of degenerations of algebraic structures (see [3]), and when one studies geometric properties of the orbits of an algebraic group ([21], [22]), and in the theory of unipotent classes of semisimple Lie groups $([29])$.

We note that since the closure of $G \cdot v$ is $G$-stable, the problem above is equivalent to deciding whether the orbit $G \cdot w$ lies in the closure of $G \cdot v$.

In this paper we review three approaches to this problem. The first one is a straightforward reduction to elimination using Gröbner bases (Section 3). The second method, due to Popov, is based on the effective Nullstellensatz (Section 4). Both these methods use an open subset of the group, isomorphic to an "easy" affine algebraic set. This is described in Section 2. The third method is specific for reductive groups that are constructed as so-called $\theta$-groups (Section $5)$. Throughout we assume that the base field is algebraically closed and of characteristic 0, as many constructions that we use (e.g., the correspondence between a linear algebraic group and its Lie algebra) only work well in characteristic 0 .

We illustrate the methods by examples. We also comment on the practical usefulness of them. Although it is possible to compute some instances of the problem using the first method, the use of Gröbner bases makes it very difficult to use it for larger examples. The second method elegantly reduces the problem to a system of linear equations. However, the number of unknowns in these equations is so large that it is questionable whether this method can be used for any nontrivial instance of the problem. The third method is rather efficient, and can be used to compute many nontrivial cases. However, it is only applicable to $\theta$-groups. 
The main ideas underlying the algorithms presented here, are all taken from sources in the literature. However, the examples that we computed have not appeared elsewhere. Also we remark that in some of the examples we report running times of the various programs used; these have been obtained on a 3.16 $\mathrm{GHz}$ machine with $32 \mathrm{~GB}$ of memory.

\section{Notation and Terminology}

Throughout we use standard terminology of algebraic geometry (see [27]) and linear algebraic groups (see [15]). In particular, by $\mathbb{A}^{n}$ we denote the $n$-dimensional affine space. By a closed set we mean the zero locus in some $\mathbb{A}^{n}$ of a set of polynomials in $n$ indeterminates. By $k$ we will denote an algebraically closed field of characteristic 0. A linear algebraic group is a subgroup of $\operatorname{GL}(n, k)$ given as the zero locus of a set of polynomials in the matrix entries. If $G$ is an algebraic group, then $\mathfrak{g}$ will denote its Lie algebra.

\section{An Embedding}

Let $G \subset \mathrm{GL}(n, k)$ be an algebraic group. Here we describe a method to obtain a closed set $X$ in an affine space, together with a regular map $\sigma: X \rightarrow G$ such that the image of $\sigma$ contains a dense and open subset of $G$. The closed set $X$ will be given by "easy" polynomial equations, so that it will be straightforward to compute in the coordinate ring $k[X]$ of $X$. The use of such an embedding for algorithmic purposes was first proposed in [26].

Lemma 1. Let $G \subset \operatorname{GL}(n, k)$ be a connected algebraic group with Lie algebra $\mathfrak{g}$. Suppose that there are algebraic subalgebras $\mathfrak{g}_{1}, \mathfrak{g}_{2}$ of $\mathfrak{g}$ such that $\mathfrak{g}$ is the direct sum of vector spaces, $\mathfrak{g}=\mathfrak{g}_{1} \oplus \mathfrak{g}_{2}$. Let $G_{1}, G_{2}$ be the connected subgroups of $G$ with Lie algebras $\mathfrak{g}_{1}, \mathfrak{g}_{2}$ respectively. Furthermore, let $X_{1}, X_{2}$ be closed sets in two affine spaces, and let $\phi_{i}: X_{i} \rightarrow G_{i}$ be regular maps. Let $x_{i} \in X_{i}$ and suppose that the differential of $\phi_{i}$ at $x_{i},\left(\mathrm{~d} \phi_{i}\right)_{x_{i}}: T_{x_{i}}\left(X_{i}\right) \rightarrow \mathfrak{g}_{i}$ is surjective, for $i=1,2$. Define $\phi: X_{1} \times X_{2} \rightarrow G$ by $\phi\left(y_{1}, y_{2}\right)=\phi_{1}\left(y_{1}\right) \phi_{2}\left(y_{2}\right)$, for $y_{i} \in X_{i}$. Then the differential of $\phi$ at $\left(x_{1}, x_{2}\right)$ is surjective.

Proof. Note that $\phi$ is the composition of the maps $X_{1} \times X_{2} \rightarrow G_{1} \times G_{2}$, given by $\left(y_{1}, y_{2}\right) \mapsto\left(\phi_{1}\left(y_{1}\right), \phi_{2}\left(y_{2}\right)\right)$ and $G_{1} \times G_{2} \rightarrow G$, given by $\left(g_{1}, g_{2}\right) \mapsto g_{1} g_{2}$. The differential of the latter map is $(x, y) \mapsto x+y$. This implies the lemma.

\subsection{Unipotent Case}

Let $U \subset \operatorname{GL}(n, k)$ be a unipotent algebraic group with Lie algebra $\mathfrak{u} \subset \mathfrak{g l}(n, k)$. Let $u_{1}, \ldots, u_{s}$ be a basis of $\mathfrak{u}$. Let $\alpha_{1}, \ldots, \alpha_{s} \in k$, then $\exp \left(\sum_{i=1}^{s} \alpha_{i} u_{i}\right)$ lies in $U$. Moreover, the map $\mathbb{A}^{s} \rightarrow U$ given by $\left(\alpha_{1}, \ldots, \alpha_{s}\right) \mapsto \exp \left(\sum_{i=1}^{s} \alpha_{i} u_{i}\right)$ is bijective and regular (see [4], Ch. V, §3, No. 4, Proposition 14). Its differential (at the point $(0, \ldots, 0))$ is the map $\left(\alpha_{1}, \ldots, \alpha_{s}\right) \mapsto \sum_{i=1}^{s} \alpha_{i} u_{i}$. 


\subsection{Diagonalisable Case}

For a subset $E \subset \mathbb{Z}^{n}$ set

$$
T_{E}=\left\{\operatorname{diag}\left(\alpha_{1}, \ldots, \alpha_{n}\right) \in k^{n} \mid \prod_{i=1}^{n} \alpha_{i}^{e_{i}}=1 \text { for all } e=\left(e_{1}, \ldots, e_{n}\right) \in E\right\}
$$

Then $T_{E}$ is an algebraic subgroup of $\operatorname{GL}(n, k)$, with Lie algebra

$$
\mathfrak{t}_{E}=\left\{\operatorname{diag}\left(\alpha_{1}, \ldots, \alpha_{n}\right) \in k^{n} \mid \sum_{i=1}^{n} e_{i} \alpha_{i}=0 \text { for all } e=\left(e_{1}, \ldots, e_{n}\right) \in E\right\} .
$$

It is clear that $T_{E}=T_{\Lambda}$, where $\Lambda \subset \mathbb{Z}^{n}$ is the lattice generated by $E$.

Now let $T \subset \operatorname{GL}(n, k)$ be an algebraic group consisting of diagonal matrices. Then there is an $E \subset \mathbb{Z}^{n}$ such that $T=T_{E}$ (see [30], §22.5). So, if $\mathfrak{t} \subset \mathfrak{g l}(n, k)$ is an algebraic Lie algebra consisting of diagonal matrices, then there is an $E \subset \mathbb{Z}^{n}$ with $\mathfrak{t}=\mathfrak{t}_{E}$. Let $\Lambda_{\mathfrak{t}} \subset \mathbb{Z}^{n}$ be the lattice defined by

$$
\Lambda_{\mathfrak{t}}=\left\{\left(e_{1}, \ldots, e_{n}\right) \in \mathbb{Z}^{n} \mid \sum_{i=1}^{n} e_{i} \alpha_{i}=0 \text { for all } \operatorname{diag}\left(\alpha_{1}, \ldots, \alpha_{n}\right) \in \mathfrak{t}\right\}
$$

then $\mathfrak{t}=\mathfrak{t}_{E}$, where $E$ is a basis of $\Lambda_{\mathfrak{t}}$. Moreover, $\mathfrak{t}$ is the Lie algebra of the group $T_{E}$.

Remark 2. Concerning the problem to compute a basis of $\Lambda_{\mathfrak{t}}$ we remark the following. Of course it suffices to have $\sum_{i=1}^{n} e_{i} \alpha_{i}=0$, where $\operatorname{diag}\left(\alpha_{1}, \ldots, \alpha_{n}\right)$ runs through a basis of $\mathfrak{t}$. If the $\alpha_{i}$ lie in a fixed number field $K$, then this leads to linear equations for the $e_{i}$ with coefficients in $\mathbb{Q}$ (after expressing the $\alpha_{i}$ as linear combinations of a fixed basis of $K$ over $\mathbb{Q}$ ). Then a basis of $\Lambda_{\mathfrak{t}}$ can be found by the saturation algorithm. The paper [11] contains a description of such an algorithm, based on the Smith normal form algorithm. MAGMA ([2]) contains an implementation of a different algorithm for computing saturations.

Let $E=\left\{e^{1}, \ldots, e^{r}\right\}$ be a basis of $\Lambda_{\mathfrak{t}}$. Let $L \subset \mathbb{Z}^{n}$ be the lattice consisting of all $d=\left(d_{1}, \ldots, d_{n}\right)$ such that $\sum_{i} d_{i} e_{i}^{k}=0$ for $1 \leq k \leq r$. Let $\left\{d^{1}, \ldots, d^{s}\right\}$ be a basis of $L$. Define $\psi_{j}: k^{*} \rightarrow T_{E}$ by $\psi_{j}(t)=\operatorname{diag}\left(t^{d_{1}^{j}}, \ldots, t^{d_{n}^{j}}\right)$. Then the differential of $\psi_{j}$ at 1 maps $\alpha \in k$ to $\alpha \operatorname{diag}\left(d_{1}^{j}, \ldots, d_{n}^{j}\right)$. Now consider the map $\psi:\left(k^{*}\right)^{s} \rightarrow T_{E}$, given by $\psi\left(t_{1}, \ldots, t_{s}\right)=\psi_{1}\left(t_{1}\right) \cdots \psi_{s}\left(t_{s}\right)$. By Lemma 1 its differential at the point $(1, \ldots, 1)$ is surjective.

Finally, let $\widetilde{T} \subset \mathrm{GL}(n, k)$ be a diagonalisable connected algebraic group, i.e., there is an $A \in \operatorname{GL}(n, k)$ such that $T=A \widetilde{T} A^{-1}$ consists of diagonal matrices. By the construction above we get a regular map $\psi:\left(k^{*}\right)^{s} \rightarrow T$ with a surjective differential. So also the differential of $\tilde{\psi}:\left(k^{*}\right)^{s} \rightarrow \widetilde{T}, \tilde{\psi}(a)=A^{-1} \psi(a) A$ is surjective. 


\subsection{General Case}

Let $G \subset \operatorname{GL}(n, k)$ be a connected algebraic group, with Lie algebra $\mathfrak{g} \subset \mathfrak{g l}(n, k)$. Let $\mathfrak{s}$ denote the solvable radical of $\mathfrak{g}$, and $\mathfrak{l}$ a Levi subalgebra of $\mathfrak{g}$, and $\mathfrak{n}$ the largest ideal of $\mathfrak{g}$ consisting of nilpotent elements. Then by [4], Chapter V, §4, Proposition 5 (see also [24]), $\mathfrak{s}$ has a commutative subalgebra $\mathfrak{d}$ consisting of semisimple elements, with the following properties

1. $\mathfrak{s}=\mathfrak{d}+\mathfrak{n}$ (semidirect sum),

2. $[\mathfrak{l}, \mathfrak{d}]=0$,

3. $\mathfrak{n}$ is the set consisting of all nilpotent elements of $\mathfrak{s}$.

There exist algorithms to obtain bases of $\mathfrak{s}$ and $\mathfrak{l}$, cf. [10]. Moreover, in [11] algorithms are described for obtaining bases of $\mathfrak{d}, \mathfrak{n}$. Let $\mathfrak{h}$ be a Cartan subalgebra of $\mathfrak{l}$, and $\mathfrak{n}^{+}, \mathfrak{n}^{-}$the sums of the positive, respectively negative, root spaces with respect to $\mathfrak{h}$ (relative to a choice of positive roots). Then

$$
\mathfrak{g}=\mathfrak{n}^{-} \oplus \mathfrak{h} \oplus \mathfrak{n}^{+} \oplus \mathfrak{d} \oplus \mathfrak{n} .
$$

All these subalgebras are algebraic. Furthermore, they either consist of nilpotent elements, or are diagonalisable. So by applying the constructions of Sections 2.1, 2.2 , along with Lemma 1 , we get

- an affine space $\mathbb{A}^{2 r+s}$, with coordinate ring $k\left[x_{1}, \ldots, x_{r}, y_{1}, \ldots, y_{r}, z_{1}, \ldots, z_{s}\right]$,

- a closed set $X^{r, s}$ of $\mathbb{A}^{2 r+s}$, given by the equations $x_{i} y_{i}=1$ for $1 \leq i \leq r$,

- a regular map $\sigma: X^{r, s} \rightarrow G$, and a point $x$ of $X^{r, s}$ such that the differential of $\sigma$ at $x$ maps $T_{x}\left(X^{r, s}\right)$ surjectively onto $\mathfrak{g}$.

The last statement implies that the image of $\sigma$ contains a nonempty open subset of $G$ (since the image of $\sigma$ contains an open subset of its closure (see [27], §I.5.3, Theorem 6) which is $G$ ).

Remark 3. Suppose that $G$ and hence $\mathfrak{g}$ are defined over the subfield $K$ of $k$ (e.g, over $\mathbb{Q}$ ). Then it can happen that the Cartan subalgebra $\mathfrak{h}$ is not split over $K$. In that case the image of $\sigma$ will not be defined over $K$, but over a finite extension of $K$.

Example 4. In this example we give a basis of an algebraic and reductive Lie subalgebra of $\mathfrak{g l}(6, k$. For this the $6 \times 6$-matrix with a 1 on position $(i, j)$ and zeros elsewhere will be denoted $e_{i, j}$.

Let $\mathfrak{g}=\mathfrak{n}^{-} \oplus \mathfrak{t} \oplus \mathfrak{n}^{+} \subset \mathfrak{g l}(6, k)$, where $\mathfrak{t}$ is diagonalisable (in fact, diagonal) and spanned by

$$
\begin{array}{r}
-e_{3,3}-e_{4,4}+e_{5,5}+e_{6,6},-e_{1,1}-e_{2,2}+e_{3,3}+e_{4,4}, 2 e_{1,1}+e_{3,3}-e_{4,4}+e_{5,5}-e_{6,6}, \\
2 e_{2,2}-e_{3,3}+e_{4,4}-e_{5,5}+e_{6,6},
\end{array}
$$

$\mathfrak{n}^{+}$is spanned by

$$
x_{\alpha_{1}}=-e_{5,3}-e_{6,4}, x_{\alpha_{2}}=-e_{3,1}-e_{4,2}, x_{\alpha_{3}}=-e_{5,1}-e_{6,2},
$$


and $\mathfrak{n}^{-}$is spanned by

$$
x_{-\alpha_{1}}=-e_{3,5}-e_{4,6}, x_{-\alpha_{2}}=-e_{1,3}-e_{2,4}, x_{-\alpha_{3}}=-e_{1,5}-e_{2,6} .
$$

This Lie algebra is reductive, with semisimple part of type $A_{2}$. The $x_{ \pm \alpha_{i}}$ are the root vectors, with $\alpha_{3}=\alpha_{1}+\alpha_{2}$.

We apply the construction of Section 2.2 to $\mathfrak{t}$. Here we get that $\Lambda_{\mathfrak{t}}$ is spanned by

$$
(1,-1,0,0,-1,1),(0,0,1,-1,-1,1) .
$$

Furthermore, a basis of $L$ (the lattice perpendicular to $\Lambda_{\mathfrak{t}}$ ) consists of

$$
(1,0,0,-1,0,-1),(0,1,0,1,0,1),(0,0,1,1,0,0),(0,0,0,0,1,1) .
$$

Consider the affine space $\mathbb{A}^{8}$ with coordinate ring $k\left[x_{1}, \ldots, x_{4}, y_{1}, \ldots, y_{4}\right]$. Let $X_{\mathfrak{t}}$ be the closed set in $\mathbb{A}^{8}$ given by $x_{i} y_{i}=1$ for $1 \leq i \leq 4$. Then we get the map $\psi: X_{\mathfrak{t}} \rightarrow T$ (where $T \subset \mathrm{GL}(6, k)$ is the connected algebraic group with Lie algebra $\mathfrak{t}$ ), given by

$$
\psi\left(t_{1}, \ldots, t_{4}, s_{1}, \ldots, s_{4}\right)=\operatorname{diag}\left(t_{1}, t_{2}, t_{3}, s_{1} t_{2} t_{3}, t_{4}, s_{1} t_{2} t_{4}\right) .
$$

Let $N^{+}$(respectively $N^{-}$) be the connected algebraic subgroup of GL $(6, k)$ with Lie algebra $\mathfrak{n}^{+}$(respectively, $\left.\mathfrak{n}^{-}\right)$. Using the construction of Section 2.1 we get maps $\phi_{+}: \mathbb{A}^{3} \rightarrow N^{+}, \phi_{-}: \mathbb{A}^{3} \rightarrow N^{-}$given by

$$
\begin{aligned}
\phi_{+}\left(u_{1}, u_{2}, u_{3}\right) & =\exp \left(u_{1}\left(-e_{5,3}-e_{6,4}\right)+u_{2}\left(-e_{3,1}-e_{4,2}\right)+u_{3}\left(-e_{5,1}-e_{6,2}\right)\right) \\
& =\left(\begin{array}{cccccc}
1 & 0 & 0 & 0 & 0 & 0 \\
0 & 1 & 0 & 0 & 0 & 0 \\
-u_{2} & 0 & 1 & 0 & 0 & 0 \\
0 & -u_{2} & 0 & 1 & 0 & 0 \\
\frac{1}{2} u_{1} u_{2}-u_{3} & 0 & -u_{1} & 0 & 1 & 0 \\
0 & \frac{1}{2} u_{1} u_{2}-u_{3} & 0 & -u_{1} & 0 & 1
\end{array}\right)
\end{aligned}
$$

and

$$
\begin{aligned}
\phi_{-}\left(v_{1}, v_{2}, v_{3}\right)= & \exp \left(v_{1}\left(-e_{3,5}-e_{4,6}\right)+v_{2}\left(-e_{1,3}-e_{2,4}\right)+v_{3}\left(-e_{1,5}-e_{2,6}\right)\right) \\
= & \left(\begin{array}{cccccc}
1 & 0 & -v_{2} & 0 & \frac{1}{2} v_{1} v_{2}-v_{3} & 0 \\
0 & 1 & 0 & -v_{2} & 0 & \frac{1}{2} v_{1} v_{2}-v_{3} \\
0 & 0 & 1 & 0 & -v_{1} & 0 \\
0 & 0 & 0 & 1 & 0 & -v_{1} \\
0 & 0 & 0 & 0 & 1 & 0 \\
0 & 0 & 0 & 0 & 0 & 1
\end{array}\right) .
\end{aligned}
$$

Denote the coordinate ring of $k\left[\mathbb{A}^{14}\right]$ by $k\left[x_{1}, \ldots, x_{4}, y_{1}, \ldots, y_{4}, z_{1}, \ldots, z_{6}\right]$. Let $X^{4,6} \subset \mathbb{A}^{14}$ be given by the equations $x_{i} y_{i}=1, i=1, \ldots, 4$. Then we get the map $\sigma: X^{4,6} \rightarrow G$, given by

$$
\begin{aligned}
& \sigma\left(t_{1}, \ldots, t_{4}, s_{1}, \ldots, s_{4}, u_{1}, u_{2}, u_{3}, v_{1}, v_{2}, v_{3}\right)= \\
& \phi_{-}\left(v_{1}, v_{2}, v_{3}\right) \psi\left(t_{1}, \ldots, t_{4}, s_{1}, \ldots, s_{4}\right) \phi_{+}\left(u_{1}, u_{2}, u_{3}\right) .
\end{aligned}
$$

It has the property that its image contains a non-empty open subset of $G$. 


\section{Computing the Closure by Gröbner Elimination}

Here we show how polynomial equations defining the closure of an orbit can be computed by a technique based on Gröbner bases. To the best of our knowledge, this approach was first described by Popov in [26].

Let $G \subset \operatorname{GL}(n, k)$ be a connected algebraic group with Lie algebra $\mathfrak{g} \subset$ $\mathfrak{g l}(n, k)$. Let $X^{r, s} \subset \mathbb{A}^{2 r+s}$ and $\sigma: X^{r, s} \rightarrow G$ be as in Section 2. Let $V=k^{n}$ be the space on which $G$ acts, and let $v \in V$. Let $Y \subset V$ be the closure of $G \cdot v$. Define a map $\sigma_{v}: X^{r, s} \rightarrow Y$ by $\sigma_{v}(x)=\sigma(x) \cdot v$. Then $\sigma_{v}$ is a regular map, and its image contains a nonempty open subset of $Y$. So the closure of the image of $\sigma_{v}$ is $Y$.

Defining equations for the closure of the image of a regular map can be computed using elimination techniques based on Gröbner bases (see, for example, [5]). In the situation considered here this works as follows. Let $e_{1}, \ldots, e_{n}$ be a fixed basis of $V$. Write $\sigma_{v}(x)=\sum_{i=1}^{n} \sigma_{v}^{i}(x) e_{i}$. Then $\sigma_{v}^{i}: X^{r, s} \rightarrow k$ is a regular map, i.e., it is the restriction of a polynomial in $k\left[x_{1}, \ldots, x_{r}, y_{1}, \ldots, y_{r}, z_{1}, \ldots, z_{s}\right]$ to $X^{r, s}$; we denote this polynomial also by $\sigma_{v}^{i}$. Now let $T_{1}, \ldots, T_{n}$ be auxiliary indeterminates, and let $R$ be the polynomial ring over $k$ with the indeterminates $x_{i}, y_{i}(1 \leq i \leq r), z_{j}(1 \leq j \leq s)$ and $T_{m}(1 \leq m \leq n)$. Let $I$ be the ideal of $R$ generated by $x_{i} y_{i}-1$ for $1 \leq i \leq r$ and $T_{m}-\sigma_{v}^{m}$ for $1 \leq m \leq n$. Use a monomial order relative to which any monomial involving at least one of $x_{i}, y_{i}$ or $z_{j}$ is bigger than any monomial involving only the indeterminates $T_{m}$. Let $\mathcal{G}$ be a Gröbner basis of $I$ with respect to such an order. Then $\mathcal{G} \cap k\left[T_{1}, \ldots, T_{n}\right]$ is a Gröbner basis of $J=I \cap k\left[T_{1}, \ldots, T_{n}\right]$. Moreover, the closed set in $V$ defined by $J$ is $Y$.

Example 5. Let the notation be as in Example 4. Let $v=e_{5}+e_{6}$ (where $e_{i}$ denotes the $i$-th elementary basis element of $\left.k^{6}\right)$. Then $\sigma\left(t_{1}, \ldots, t_{4}, s_{1}, \ldots, s_{4}, u_{1}, u_{2}, u_{3}, v_{1}, v_{2}, v_{3}\right)$. $v$ is

$$
\left(t_{4}\left(\frac{1}{2} v_{1} v_{2}-v_{3}\right), s_{1} t_{2} t_{4}\left(\frac{1}{2} v_{1} v_{2}-v_{3}\right),-t_{4} v_{1},-s_{1} t_{2} t_{4} v_{1}, t_{4}, s_{1} t_{2} t_{4}\right) .
$$

Let $I$ be the ideal constructed as above. MAGMA computes a Gröbner basis $\mathcal{G}$ of $I$, with respect to an elimination order, in 0.02 seconds. The intersection of $\mathcal{G}$ with $k\left[T_{1}, \ldots, T_{6}\right]$ is

$$
\left\{T_{2} T_{3}-T_{1} T_{4}, T_{2} T_{5}-T_{1} T_{6}, T_{4} T_{5}-T_{3} T_{6}\right\}
$$

So the closure $Y$ of $G \cdot v$ is the set of $\sum_{i=1}^{6} \alpha_{i} e_{i}$ such that $\alpha_{2} \alpha_{3}=\alpha_{1} \alpha_{4}$, and so on. We see that $e_{5}$ and $e_{6}$ lie in $Y$, so their orbits do as well. On the other hand, $e_{4}+e_{5}$ does not lie in $Y$.

\section{Popov's Algorithm}

In this section we describe an algorithm due to V. L. Popov ([26]). It is a method for deciding whether a given $w$ lies in the closure of $G \cdot v$. 


\subsection{Conical Groups}

We say that an algebraic group $G \subset \mathrm{GL}(n, k)$ is conical if $\lambda I_{n} \in G$ for all nonzero $\lambda \in k$, where $I_{n}$ is the $n \times n$-identity matrix. If $G$ is conical, then also an orbit $G \cdot v$ is conical, i.e., $w \in G \cdot v$ if and only if $\lambda w \in G \cdot v$ for all nonzero $\lambda \in k$.

Now let $G \subset \operatorname{GL}(n, k)$ be an algebraic group. Set $V=k^{n}$, the natural $G$ module. Let $V_{0}=k^{n+1}$ and write elements of $V_{0}$ as $\left(\alpha_{0}, v\right)$, where $\alpha_{0} \in k$ and $v \in V$. A $g \in G$ acts on $V_{0}$ by $g \cdot\left(\alpha_{0}, v\right)=\left(\alpha_{0}, g \cdot v\right)$. By $G_{0}$ we denote the corresponding image of $G$ in $\operatorname{GL}\left(V_{0}\right)$. Let $I_{V_{0}}$ be the identity endomorphism of $V_{0}$ and let $D_{0}=\left\{\lambda I_{V_{0}} \mid \lambda \in k, \lambda \neq 0\right\}$. Set $\widetilde{G}=G_{0} D_{0}$. Then $\widetilde{G}$ is an algebraic subgroup of $\mathrm{GL}\left(V_{0}\right)$.

Lemma 6. Let $v \in V$. A $w$ in $V$ lies in the closure of $G \cdot v$ if and only if $(1, w)$ lies in the closure of $\widetilde{G} \cdot(1, v)$.

Proof. Let $I$ be the vanishing ideal of $G \cdot v$ in $k\left[x_{1}, \ldots, x_{n}\right]$, and $J$ the vanishing ideal of $\widetilde{G} \cdot(1, v)$ in $k\left[x_{0}, x_{1}, \ldots, x_{n}\right]$. Since the latter orbit is conical, $J$ is spanned by homogeneous polynomials. By $k\left[x_{1}, \ldots, x_{n}\right]_{\leq d}$ we denote the space of polynomials of degree at most $d$. By $k\left[x_{0}, x_{1}, \ldots, x_{n}\right]_{d}$ we denote the space of polynomials of degree $d$. Consider the maps $\gamma: k\left[x_{0}, x_{1}, \ldots, x_{n}\right]_{d} \rightarrow k\left[x_{1}, \ldots, x_{n}\right]_{\leq d}$, $\delta: k\left[x_{1}, \ldots, x_{n}\right]_{\leq d} \rightarrow k\left[x_{0}, x_{1}, \ldots, x_{n}\right]_{d}$ given by $\gamma(f)=f\left(1, x_{1}, \ldots, x_{n}\right), \delta(g)=$ $x_{0}^{d} g\left(x_{1} / x_{0}, \ldots, x_{n} / x_{0}\right)$. These are inverses of each other. Set $I_{\leq d}=I \cap k\left[x_{1}, \ldots, x_{n}\right]_{\leq d}$ and $J_{d}=J \cap k\left[x_{0}, x_{1}, \ldots, x_{n}\right]_{d}$. It is straightforward to see that $\gamma\left(J_{d}\right) \subseteq I_{\leq d}$, $\delta\left(I_{<d}\right) \subseteq J_{d}$. So in fact these inclusions are equalities. Let $w$ lie in the closure of $G \cdot v$, then $f(w)=0$ for all $f \in I$. Let $h \in J_{d}$, then $h=\delta(f)$ for a certain $f \in I_{\leq d}$. This implies that $h(1, w)=0$. We conclude that $(1, w)$ lies in the closure of $\widetilde{G} \cdot(1, v)$. The reverse implication is shown analogously.

We conclude that we may assume that the algebraic group, and hence the orbit, is conical.

\subsection{The Degree of $G$}

Let $X \subset \mathbb{A}^{n}$ be a closed set of dimension $\ell$. Let $\Omega \subset \mathbb{A}^{n}$ be a plane of dimension $n-\ell$, "in general position". Then the number of points of $X \cap \Omega$ is called the degree of $X$. For a closed set $Y \subset \mathbb{P}^{n}$ the concept of degree is defined in the same way (see [14]). Now suppose that the vanishing ideal of $X$ is spanned by homogeneous polynomials. Then we can also view $X$ as a closed set in $\mathbb{P}^{n-1}$. Moreover, the notions of degree of $X$, seen as closed set in $\mathbb{A}^{n}$, and degree of $X$, seen as closed set in $\mathbb{P}^{n-1}$, coincide.

A closed set $Y$ in projective space gives rise to a polynomial known as the Hilbert polynomial (see [14]). If the degree of $Y$ is $d$ and the dimension is $\ell$, then the leading term of this polynomial is $\frac{d}{\ell !} x^{\ell}$.

Bayer and Stillman ([1]) have described algorithms for computing the Hilbert series, and Hilbert polynomial of a closed set in projective space. So using that it is possible to compute the degree of such a closed set, or of a conical closed 
set in affine space. Since, by the previous subsection, we may assume that the group that we are dealing with is conical, (this implies that it is an open set of a conical closed set) we can compute the degree of the group in this way.

Example 7. Polynomial equations for the group $G$, given its Lie algebra, can be computed using the methods of [11]. For the group of Example 4 this computation, performed in MAGMA, took 19669 seconds. The result is a set of homogeneous equations defining a closed set $G^{\prime}$ in $\operatorname{End}(V)$. Furthermore, $G$ consists of all $g \in G^{\prime}$ such that $\operatorname{det}(g) \neq 0$. The Hilbert polynomial of $G^{\prime}$ is then computed in 0.000 seconds. It is

$$
\begin{array}{r}
\frac{1}{403200} X^{10}+\frac{83}{725760} X^{9}+\frac{23}{10080} X^{8}+\frac{625}{24192} X^{7}+\frac{1171}{6400} X^{6}+\frac{29111}{34560} X^{5}+ \\
\frac{102293}{40320} X^{4}+\frac{177911}{36288} X^{3}+\frac{291197}{50400} X^{2}+\frac{9397}{2520} X+1 .
\end{array}
$$

From the leading term we see that the dimension is 10 (we knew that already), and the degree is 9 .

We see that a disadvantage of this approach is the necessity to know defining polynomials of $G$. However, if they are available from the start, then this is a good way to compute the degree.

In the case where $G$ is reductive there exists a different method based on a formula due to Kazarnovskiı ([20]). Here we do not describe this formula (for that see Kazarnovskiı's paper, or [7], Section 4.7, or [26]), but instead illustrate it for the group $G$ of Example 4 .

Example 8. Let the notation be as in Example 4. Then $T$ is a maximal torus of $G$. The elements of $T$ are

$$
t=\operatorname{diag}\left(t_{1}, t_{2}, t_{3}, t_{1}^{-1} t_{2} t_{3}, t_{4}, t_{1}^{-1} t_{2} t_{4}\right) .
$$

The characters, $\chi: T \rightarrow k^{*}$ are given by $\chi(t)=t_{1}^{d_{1}} \cdots t_{4}^{d_{4}}$, where $d_{i} \in \mathbb{Z}$. We let $X$ be the character group of $T$, written additively: so it is a free $\mathbb{Z}$-module with basis $\chi_{1}, \ldots, \chi_{4}$, where $\chi_{i}(t)=t_{i}$, and a character $\chi$ as above corresponds to $d_{1} \chi_{1}+\cdots+d_{4} \chi_{4}$. We also represent $\chi$ by the row vector $\left(d_{1}, \ldots, d_{4}\right)$. Now the basis vectors $e_{1}, \ldots, e_{6}$ of $V=k^{6}$ are weight vectors of $T$. The corresponding weights are

$$
(1,0,0,0),(0,1,0,0),(0,0,1,0),(-1,1,1,0),(0,0,0,1),(-1,1,0,1) .
$$

We let $\mathcal{P} \subset E=\mathbb{R}^{4}$ be the polytope that is the convex hull of these points along with $(0,0,0,0)$.

The group $G$ acts on $\mathfrak{g}$ via $g \cdot x=g x g^{-1}$. For this action the $x_{ \pm \alpha_{i}}$ are weight vectors of $T$, and we denote the corresponding characters by $\pm \alpha_{i}$. Then

$$
\alpha_{1}=(0,0,-1,1), \alpha_{2}=(-1,0,1,0), \alpha_{3}=(-1,0,0,1) .
$$

Let $W=N_{G}(T) / T$ be the Weyl group of $G$. This group acts on $X$, and hence on $E$. We need a $W$-invariant inner product $($,$) on E$, which we get as follows. 
We can also view the elements of $X$ as elements of the dual space of $t$. Here a $\chi \in X$ corresponds its differential (at the identity) $\mathrm{d} \chi \in \mathfrak{t}^{*}$ : for $\chi$ as above and $u \in \mathfrak{t}$ we have $\mathrm{d} \chi(u)=d_{1} u_{11}+d_{2} u_{22}+d_{3} u_{33}+d_{4} u_{55}$. As $W \cong N_{G}(\mathfrak{t}) / Z_{G}(\mathfrak{t})$, it also acts on $\mathfrak{t}$. A $W$-invariant nondegenerate positive definite bilinear form on $\mathfrak{t}$ is given by $(x, y)=\operatorname{Tr}(x y)$. Using this bilinear form we identify $\mathfrak{t}$ and its dual space. By this identification we then also get a $W$-invariant bilinear form on the dual of $\mathfrak{t}$, and hence on $E$. In the basis of the $\chi_{i}$ this form is given by the matrix

$$
\left(\begin{array}{cccc}
4 & 2 & 1 & 1 \\
2 & 4 & -1 & -1 \\
1 & -1 & 4 & 1 \\
1 & -1 & 1 & 4
\end{array}\right)
$$

Now we consider the functions $\alpha_{i}^{\vee}: E \rightarrow \mathbb{R}$ given by $\alpha_{i}^{\vee}(v)=\frac{2\left(v, \alpha_{i}\right)}{\left(\alpha_{i}, \alpha_{i}\right)}$. Let $v=$ $\sum_{i} d_{i} \chi_{i}$. Then $\alpha_{1}^{\vee}(v)=-d_{3}+d_{4}, \alpha_{2}^{\vee}(v)=-d_{1}-d_{2}+d_{3}, \alpha_{3}^{\vee}(v)=-d_{1}-d_{2}+d_{4}$. Let $I$ be the value of the integral of the function $\left(\alpha_{1}^{\vee}\right)^{2}\left(\alpha_{2}^{\vee}\right)^{2}\left(\alpha_{3}^{\vee}\right)^{2}$ over $\mathcal{P}$. The package LATTE INTEGRALE ([6]) can perform this integration. After 0.01 seconds the program returned $I=\frac{1}{16800}$.

Now let $m_{1}, \ldots, m_{r}$ be the exponents of $W$ (see [16], §3.16). Here the root system is of type $A_{2}$, and therefore $r=2, m_{1}=1, m_{2}=2$. According to Kazarnovskiı̌'s formula we have

$$
\operatorname{deg}(G)=\frac{\operatorname{dim}(G) !}{|W|\left(m_{1} ! m_{2} !\right)^{2}} I=9 .
$$

\subsection{The Algorithm}

Here we let $G \subset \mathrm{GL}(n, k)$ be a conical algebraic group. By $d$ we denote its degree. Let $\sigma: X^{r, s} \rightarrow G$ be an embedding as constructed in Section 2. Here $X^{r, s} \subset$ $\mathbb{A}^{2 r+s}$, and the coordinate ring of $X^{r, s}$ is $R_{r, s}=k\left[x_{1}, \ldots, x_{r}, y_{1}, \ldots, y_{r}, z_{1}, \ldots, z_{s}\right] / I_{r, s}$, where $I_{r, s}$ is generated by $x_{i} y_{i}-1,1 \leq i \leq r$. Note that a basis of $R_{r, s}$ is given by the set of monomials $x_{1}^{k_{1}} \cdots x_{r}^{k_{r}} z_{1}^{m_{1}} \cdots z_{s}^{m_{s}}$, with $k_{i} \in \mathbb{Z}$ and $m_{i} \in \mathbb{N}$ (here we write $x_{i}^{-1}$ in place of $y_{i}$ ). So it is straightforward to compute in the ring $R_{r, s}$.

Let $v \in k^{n}$, and let $Y$ be the closure of the orbit $G \cdot v$. Then we consider the regular map $\psi: X^{r, s} \rightarrow Y$ given by $\psi(a)=\sigma(a) \cdot v$. Since the image of $\sigma$ contains a dense and open subset of $G$, also the image of $\psi$ is dense in $Y$. Let $k\left[T_{1}, \ldots, T_{n}\right]$ be the coordinate ring of $k^{n}$. We view the elements of $k\left[T_{1}, \ldots, T_{n}\right]$ as functions $k^{n} \rightarrow k$ (i.e., by fixing a basis of $k^{n}$ ), and let $\bar{T}_{i}$ be the restriction of $\bar{T}_{i}$ to $Y$. Let $\psi^{*}: k[Y] \rightarrow R_{r, s}$ be the comorphism of $\psi$ (i.e., $\left.\psi^{*}(f)(a)=f(\psi(a))\right)$. Set $S_{i}=\psi^{*}\left(\bar{T}_{i}\right)$.

Theorem 9. Let $w \in k^{n}$ and write $w=\left(w_{1}, \ldots, w_{n}\right)$. Let $j_{1}, \ldots, j_{m}$ be the indices such that $\bar{T}_{j_{k}} \neq 0$. Suppose that $w_{i}=0$ if $i \notin\left\{j_{1}, \ldots, j_{m}\right\}$. Then $w \notin Y$ if and only if there are $F_{1}, \ldots, F_{m} \in k\left[Z_{1}, \ldots, Z_{m}\right]$ such that $\operatorname{deg}\left(F_{i}\right) \leq 2 d-2$ and

$$
\sum_{k=1}^{m}\left(S_{j_{k}}-w_{j_{k}}\right) F_{k}\left(S_{j_{1}}, \ldots, S_{j_{m}}\right)=1
$$


Proof. (Sketch) Note that $w \notin Y$ if and only if there is no point of $Y$ where the functions $\bar{T}_{j_{k}}-w_{j_{k}}$ simultaneously vanish. By Hilbert's Nullstellensatz this is equivalent to the existence of $f_{1}, \ldots, f_{m} \in k[Y]$ such that $\sum_{i=1}^{m}\left(\bar{T}_{j_{k}}-w_{j_{k}}\right) f_{k}=1$. Now the effective Nullstellensatz (see [17]) strengthens this by also giving a bound on the degrees of the $f_{i}$. Here the bound turns out to be $\operatorname{deg}\left(\left(\bar{T}_{j_{k}}-\right.\right.$ $\left.\left.w_{j_{k}}\right) f_{k}\right) \leq 2 \operatorname{deg}(X)-1$.

The degree of an $f \in k[Y]$ is the minimum degree of an $\hat{f} \in k\left[T_{1}, \ldots, T_{n}\right]$ whose restriction to $Y$ equals $f$. Since $Y$ is irreducible and conical we have that $\operatorname{deg}(f g)=\operatorname{deg}(f)+\operatorname{deg}(g)$ for all $f, g \in k[Y]$. Moreover, $\operatorname{deg}\left(\bar{T}_{j_{k}}\right)=1$ for $1 \leq k \leq m$.

Also, since $Y$ is (the closure of) the image of $G$ under the map $\operatorname{End}(V) \rightarrow V$, $g \mapsto g \cdot v$, we have $\operatorname{deg}(Y) \leq \operatorname{deg}(G)=d$ (see [7], Proposition 4.7.10).

It follows that we get the bound $\operatorname{deg}\left(f_{k}\right) \leq 2 d-2$. Now mapping the equality $\sum_{i=1}^{m}\left(\bar{T}_{j_{k}}-w_{j_{k}}\right) f_{k}=1$ with $\psi^{*}$ to $R_{r, s}$, we get the statement of the lemma. Note that $\psi^{*}$ is injective, as the image of $\psi$ contains a dense open subset of $Y$.

The idea of the algorithm is to write $F_{1}, \ldots, F_{m} \in k\left[Z_{1}, \ldots, Z_{m}\right]$ of degree $2 d-2$, with unknowns as coefficients. Then the condition in the theorem yields a set of linear equations for these unknowns, since we have a basis of $R_{r, s}$. Then $w \in Y$ if and only if these equations have no solution. So the number of unknowns in the equations that we get is

$$
m\left(\begin{array}{c}
m+2 d-2 \\
m
\end{array}\right) .
$$

The number of equations is equal to the number of different monomials in $R_{r, s}$ that appear when evaluating a monomial of $k\left[Z_{1}, \ldots, Z_{m}\right]$, of degree $\leq 2 d-2$, in $S_{1}, \ldots, S_{m}$.

Example 10. We let the notation be as in Example 4. As seen in Examples 7, 8, here we have $d=9$. With $v$ as in Example 5 we get $m=6$. So the number of unknowns in this case will be 447678 .

Remark 11. It is also possible to base a different algorithm on Theorem 9. Let $A$ be the subalgebra of $R_{r, s}$ generated by $S_{j_{1}}, \ldots, S_{j_{m}}$. By elimination based on Gröbner bases we can compute all polynomial relations among these generators. So we get an isomorphism $k\left[U_{1}, \ldots, U_{m}\right] / J \rightarrow A$. Now $w \notin X$ if and only if the ideal of $k\left[U_{1}, \ldots, U_{m}\right]$ generated by $J$ and $U_{k}-w_{j_{k}}$ contains 1 . This again can be checked by a Gröbner basis computation. However, note that computing the polynomial relations among the $S_{j_{k}}$ leads to the same computation as computing defining polynomials for the closure of $G \cdot v$, cf. Example 5 .

\section{Closures of Nilpotent Orbits of $\theta$-Groups}

The algebraic groups known as $\theta$-groups are reductive, and arise from gradings of (semi-) simple Lie algebras. They have been introduced and studied by Vinberg 
in the 70's ([31], [32], [33]). We divide this section in a number of subsections. In the first of these we sketch how $\theta$-groups come about; for more detailed information we refer to the above mentioned papers of Vinberg. The second contains some general facts on nilpotent orbits of $\theta$-groups. In the remaining sections we sketch an algorithm from [9] to decide whether a given nilpotent orbit is contained in the closure of another one.

\section{$5.1 \quad \theta$-Groups}

Let $\mathfrak{g}$ be a (semi-) simple Lie algebra over $k$. Let $G$ be the connected component of the identity of the automorphism group of $\mathfrak{g}$. This group has Lie algebra adg, which we identify with $\mathfrak{g}$. By $\mathbb{Z}_{m}$ we denote the group $\mathbb{Z} / m \mathbb{Z}$, which, by convention, is equal to $\mathbb{Z}$ if $m=\infty$. We consider $\mathbb{Z}_{m}$-gradings of $\mathfrak{g}$ :

$$
\mathfrak{g}=\bigoplus_{i \in \mathbb{Z}_{m}} \mathfrak{g}_{i}
$$

This means that the $\mathfrak{g}_{i}$ are subspaces of $\mathfrak{g}$ such that $\left[\mathfrak{g}_{i}, \mathfrak{g}_{j}\right] \subset \mathfrak{g}_{i+j}$. In particular, $\mathfrak{g}_{0}$ is a subalgebra, and it can be shown that it is reductive. Let $G_{0}$ be the connected subgroup of $G$ with Lie algebra $\mathfrak{g}_{0}$ (or, more precisely, ad $\mathfrak{g}_{0}$ ). Since $\left[\mathfrak{g}_{0}, \mathfrak{g}_{1}\right] \subset \mathfrak{g}_{1}$ we also get that $G_{0}$ stabilizes $\mathfrak{g}_{1}$. The corresponding representation of $G_{0}$ is called a $\theta$-representation, and $G_{0}$ is called a $\theta$-group.

Example 12. In this example we consider the Lie algebra of type $D_{4}$. We start by giving a multiplication table of $\mathfrak{g}$. Let $\Phi$ be the root system of type $D_{4}$, with basis of simple roots $\Delta=\left\{\beta_{1}, \beta_{2}, \beta_{3}, \beta_{4}\right\}$. The simple roots correspond to the nodes of the Dynkin diagram in the following way

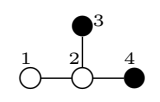

Let $Q$ denote the $\mathbb{Z}$-span of $\Delta$. We define a bilinear form $($,$) on Q$ by $\left(\beta_{i}, \beta_{i}\right)=2,\left(\beta_{i}, \beta_{j}\right)=-1$ if $i \neq j$ and $i, j$ are connected in the Dynkin diagram. Otherwise $\left(\beta_{i}, \beta_{j}\right)=0$. Also we define a group homomorphism $\varepsilon: Q \times Q \rightarrow$ $\{1,-1\}$ (where the latter is a multiplicative group), by $\varepsilon\left(\beta_{i}, \beta_{j}\right)=-1$ if $i=j$ or $i \neq j$ and $i<j$ and $i, j$ are connected in the Dynkin diagram. Otherwise $\varepsilon\left(\beta_{i}, \beta_{j}\right)=1$. Also we define $\tilde{\varepsilon}: \Phi \times \Phi \rightarrow\{1,-1$,$\} by \tilde{\varepsilon}\left(\beta, \beta^{\prime}\right)=\varepsilon\left(\beta, \beta^{\prime}\right)$ if both $\beta$ and $\beta^{\prime}$ are positive, or exactly one of them is positive and $\beta+\beta^{\prime}$ is negative. Otherwise $\tilde{\varepsilon}\left(\beta, \beta^{\prime}\right)=-\varepsilon\left(\beta, \beta^{\prime}\right)$.

Let $\mathfrak{g}$ be the 28 -dimensional vector space spanned by $h_{1}, \ldots, h_{4}$, along with $x_{\beta}$ for $\beta \in \Phi$. On $\mathfrak{g}$ we define the Lie bracket by

$$
\begin{aligned}
{\left[h_{i}, h_{j}\right] } & =0 \\
{\left[h_{i}, x_{\beta}\right] } & =\left(\beta, \alpha_{i}\right) x_{\beta} \\
{\left[x_{\beta}, x_{-\beta}\right] } & =h_{\beta} \\
{\left[x_{\beta}, x_{\beta^{\prime}}\right] } & =\tilde{\varepsilon}\left(\beta, \beta^{\prime}\right) x_{\beta+\beta^{\prime}},
\end{aligned}
$$


where we set $h_{\beta}=\sum_{i=1}^{4} m_{i} h_{i}$ for $\beta=\sum_{i=1}^{4} m_{i} \beta_{i}$, and $x_{\gamma}=0$ if $\gamma \in Q \backslash \Phi$. With this Lie bracket, $\mathfrak{g}$ is a simple Lie algebra of type $D_{4}$ (cf. [18], Proposition 7.8, and also [10], Proposition 5.13.4). (We use $\tilde{\varepsilon}$ instead of $\varepsilon$ because in the mentioned references the relation $\left[x_{\beta}, x_{-\beta}\right]=-h_{\beta}$ is used.)

Next we define a grading on $\mathfrak{g}$. The simple roots corresponding to the black nodes in the Dynkin diagram have degree 1 , the others have degree 0 . Also, the degree of a sum of simple roots will be the sum of the degrees. Finally, the degree of a negative root $\beta$ is minus the degree of $-\beta$. Then we define a $\mathbb{Z}$-grading of $\mathfrak{g}$ by letting $\mathfrak{g}_{0}$ be equal to the span of the $h_{i}$ along with all $x_{\beta}$ such that the degree of $\beta$ is 0 . Furthermore, $\mathfrak{g}_{i}$ will be the span of all $x_{\beta}$ such that the degree of $\beta$ is $i$.

Then $\mathfrak{g}_{0}$ is the sum of a simple Lie algebra of type $A_{2}$, and a 2-dimensional torus. The space $\mathfrak{g}_{1}$ is spanned by $x_{\beta_{3}}, x_{\beta_{4}}, x_{\beta_{2}+\beta_{3}}, x_{\beta_{2}+\beta_{4}}, x_{\beta_{1}+\beta_{2}+\beta_{3}}, x_{\beta_{1}+\beta_{2}+\beta_{4}}$.

The image of $\mathfrak{g}_{0}$ in $\mathfrak{g l}(6, k)$ (corresponding to the action on $\mathfrak{g}_{1}$ ) is the Lie algebra considered in Example 4. The matrices given in that example are defined relative to the basis above.

\subsection{Nilpotent Orbits of $\theta$-Groups}

Let the notation be as in the previous subsection. Let $e \in \mathfrak{g}_{1}$. Then the orbit $G_{0} e$ is said to be nilpotent if the (Zariski-) closure of $G_{0} e$ contains 0 . We say that $e \in \mathfrak{g}_{1}$ is nilpotent if the orbit $G_{0} e$ is nilpotent. It can be shown that this is equivalent to ade being a nilpotent endomorphism of $\mathfrak{g}$.

Let $e \in \mathfrak{g}_{1}$ be nilpotent. Then there are $h \in \mathfrak{g}_{0}, f \in \mathfrak{g}_{-1}$ such that $[e, f]=h$, $[h, e]=2 e,[h, f]=-2 f$. We say that $e$ lies in the homogeneous $\mathfrak{s}_{2}$-triple $(h, e, f)$. The element $h$ is called a characteristic of $e$.

Let $e_{i} \in \mathfrak{g}_{1}$, for $i=1,2$, be nilpotent elements lying in homogeneous $\mathfrak{s l}_{2}-$ triples $\left(h_{i}, e_{i}, f_{i}\right)$. Then the following are equivalent:

- $e_{1}, e_{2}$ are $G_{0}$-conjugate,

- $\left(h_{1}, e_{1}, f_{1}\right),\left(h_{2}, e_{2}, f_{2}\right)$ are $G_{0}$-conjugate,

- $h_{1}, h_{2}$ are $G_{0}$-conjugate.

This equivalence yields an algorithm to list the nilpotent orbits of $G_{0}$ (see [12]). Alternatively, Vinberg has devised a method, using so-called carrier algebras, to list the nilpotent orbits of a $\theta$-group ([33], see also [12]). Also an algorithm by Popov ([25]) can be used to list the nilpotent orbits of a $\theta$-group. Furthermore, Kawanake ([19]) considered the question of listing the nilpotent orbits of a $\theta$-group also for base fields of characteristic $p>0$.

We let $\mathfrak{h}_{0}$ be a fixed Cartan subalgebra of $\mathfrak{g}_{0}$. Then, after acting with an element of $G_{0}$, we may assume that $h_{1}, h_{2} \in \mathfrak{h}_{0}$. Furthermore, $h_{1}, h_{2} \in \mathfrak{h}_{0}$ are $G_{0}$-conjugate if and only if they are conjugate under the Weyl group $W_{0}=$ $N_{G_{0}}\left(\mathfrak{h}_{0}\right) / Z_{G_{0}}\left(\mathfrak{h}_{0}\right)$, which is a finite group, isomorphic to the Weyl group of the root system of $\mathfrak{g}_{0}$.

Example 13. Let the notation be as in Example 12. We let $\mathfrak{h}_{0}$ be the Cartan subalgebra of $\mathfrak{g}_{0}$ spanned by $h_{1}, h_{2}, h_{3}, h_{4}$ (this is a Cartan subalgebra of $\mathfrak{g}$ as 
well). Since we deal with a $\mathbb{Z}$-grading there are only nilpotent orbits. Using the implementation of the algorithms of [12], in the SLA package ([13]) of GAP4 ([8]) we immediately get the list of nilpotent orbits, $\mathcal{O}_{1}, \ldots, \mathcal{O}_{4}$, each given by a homogeneous $\mathfrak{s l}_{2}$-triple:

$$
\begin{aligned}
& \mathcal{O}_{1}:\left(h_{1}+h_{2}+h_{3}, x_{\beta_{1}+\beta_{2}+\beta_{3}}, x_{-\beta_{1}-\beta_{2}-\beta_{3}}\right) \\
& \mathcal{O}_{2}:\left(h_{1}+h_{2}+h_{4}, x_{\beta_{1}+\beta_{2}+\beta_{4}}, x_{-\beta_{1}-\beta_{2}-\beta_{4}}\right) \\
& \mathcal{O}_{3}:\left(2 h_{1}+2 h_{2}+h_{3}+h_{4}, x_{\beta_{1}+\beta_{2}+\beta_{3}}+x_{\beta_{1}+\beta_{2}+\beta_{4}}, x_{-\beta_{1}-\beta_{2}-\beta_{3}}+x_{-\beta_{1}-\beta_{2}-\beta_{4}}\right) \\
& \mathcal{O}_{4}:\left(2 h_{1}+4 h_{2}+2 h_{3}+2 h_{4}, x_{\beta_{2}+\beta_{4}}+x_{\beta_{1}+\beta_{2}+\beta_{3}}, 2 x_{-\beta_{2}-\beta_{4}}+2 x_{-\beta_{1}-\beta_{2}-\beta_{3}}\right) \text {. }
\end{aligned}
$$

In this example $W_{0}$ is the Weyl group of type $A_{2}$, generated by $s_{i}=s_{\beta_{i}}, i=1,2$. A reflection $s_{\beta}$ acts on $\mathfrak{h}_{0}$ by $s_{\beta}(h)=h-\left(\beta, \beta_{h}\right) h_{\beta}$, where $\beta_{h}=\sum_{i=1}^{4} a_{i} \beta_{i}$ if $h=\sum_{i=1}^{4} a_{i} h_{i}$.

\subsection{The Algorithm}

We set $V=\mathfrak{g}_{1}$. Corresponding to a homogeneous $\mathfrak{s l}_{2}$-triple $(h, e, f)$ we define the spaces

$$
V_{k}(h)=\{v \in V \mid[h, v]=k v\} \text { and } V_{\geq 2}(h)=\bigoplus_{k \geq 2} V_{k}(h) .
$$

Let $\left(h^{\prime}, e^{\prime}, f^{\prime}\right),(h, e, f)$ be two homogeneous $\mathfrak{s l}_{2}$-triples. The algorithm to decide whether $G_{0} e^{\prime}$ lies in the closure of $G_{0} e$ is based on the following theorem. One of the main ingredients of the proof, which here is omitted, is the following result from [34], Theorem 5.6: the closure of $G_{0} e$ is equal to $G_{0}\left(V_{\geq 2}(h)\right)$.

Theorem 14. $G_{0} e^{\prime}$ is contained in the closure of $G_{0} e$ if and only if there is a $w \in W_{0}$ such that $U_{w}=V_{2}\left(h^{\prime}\right) \cap V_{\geq 2}(w h)$ contains a point of $G_{0} e^{\prime}$. Moreover, in that case the intersection of $U$ and $G_{0} e^{\prime}$ is open and dense in $U_{w}$.

Deciding whether a given $u \in U_{w}$ lies in $G_{0} e^{\prime}$ is straightforward: this is equivalent to the existence of $f^{\prime \prime} \in \mathfrak{g}_{-1}$ such that $\left(h^{\prime}, u, f^{\prime \prime}\right)$ is an $\mathfrak{s l}_{2}$-triple ([9], Lemma 2.6), and the latter can be established by solving a small system of linear equations.

So the previous theorem yields a straightforward algorithm:

1. For each $w \in W_{0}$ take a random element $u$ of $U_{w}$, and check whether $u \in G_{0} e^{\prime}$

2. If this holds, then $G_{0} e^{\prime}$ lies in the closure of $G_{0} e$.

3. If $u \notin G_{0} e^{\prime}$ then show that $U_{w} \cap G_{0} e^{\prime}=\emptyset$, and go to the next element of $W_{0}$.

The last step contains an algorithmic problem that we haven't solved yet. This will be the subject of the next subsection. 
Example 15. Let the notation be as in Examples 12, 13. Let $\rho: \mathfrak{g}_{0} \rightarrow \mathfrak{g l}\left(\mathfrak{g}_{1}\right)$ be the representation given by the action of $\mathfrak{g}_{0}$ on $\mathfrak{g}_{1}$. We give a $\rho(x)$ by its matrix relative to the basis of $\mathfrak{g}_{1}$ given in Example 12. Then for the characteristics of the nilpotent orbits we have

$$
\begin{aligned}
\rho\left(h_{1}+h_{2}+h_{3}\right) & =\operatorname{diag}(1,-1,1,-1,2,0) \\
\rho\left(h_{1}+h_{2}+h_{4}\right) & =\operatorname{diag}(-1,1,-1,1,0,2) \\
\rho\left(2 h_{1}+2 h_{2}+h_{3}+h_{4}\right) & =\operatorname{diag}(0,0,0,0,2,2) \\
\rho\left(2 h_{1}+4 h_{2}+2 h_{3}+2 h_{4}\right) & =\operatorname{diag}(0,0,2,2,2,2) .
\end{aligned}
$$

From this, the given representatives of the orbits in Example 13, and Theorem 14 we immediately get that $\mathcal{O}_{1}, \mathcal{O}_{2} \subset \overline{\mathcal{O}_{3}}$ and $\mathcal{O}_{3} \subset \overline{\mathcal{O}_{4}}$. The only possible inclusion relation remaining is the one between $\mathcal{O}_{1}$ and $\mathcal{O}_{2}$. They have the same dimension (3), so one cannot be contained in the closure of the other. It can also be seen using Theorem 14. The $W_{0}$-conjugates of $h_{1}+h_{2}+h_{4}$ are $h_{2}+h_{4}$ and $h_{4}$. In all cases we get that $U_{w}=0$.

\subsection{Deciding Emptiness}

Let $(h, e, f)$ be a homogeneous $\mathfrak{s l}_{2}$-triple. Let $U \subset V_{2}(h)$ be a given subspace. Here we describe an algorithm for deciding whether $U \cap G_{0} e=\emptyset$.

Consider the subgroup $Z_{0}(h)=\left\{g \in G_{0} \mid g \cdot h=h\right\}$, with Lie algebra $\mathfrak{z}_{0}(h)=\left\{x \in \mathfrak{g}_{0} \mid[x, h]=0\right\}$. From the representation theory of $\mathfrak{s l}_{2}$ it follows that ade $: \mathfrak{z}_{0}(h) \rightarrow V_{2}(h)$ is surjective. But that is the differential of the map $Z_{0}(h) \rightarrow V_{2}(h), g \mapsto g \cdot e$. It follows that $Z_{0}(h) e$ contains a nonempty open subset of $V_{2}(h)$. Moreover, $G_{0} e \cap V_{2}(h)=Z_{0}(h) e$ (see the proof of [9], Lemma 2.6). It follows that a given $v \in V_{2}(h)$ lies in $G_{0} e$ if and only if it lies in $Z_{0}(h) e$; but that happens precisely when $\operatorname{dim} Z_{0}(h) v=\operatorname{dim} V_{2}(h)$. Furthermore, $\operatorname{dim} Z_{0}(h) v=$ $\operatorname{dim}\left[\mathfrak{z}_{0}(h), v\right]$.

Now let $v_{1}, \ldots, v_{s}, x_{1}, \ldots, x_{n}$ be bases of $V_{2}(h)$ and $\mathfrak{z}_{0}(h)$ respectively. Let $\psi_{1}, \ldots, \psi_{s}$ be the dual basis of $V_{2}(h)$ (i.e., $\left.\psi_{j}\left(v_{i}\right)=\delta_{i, j}\right)$. For a $v \in V$ we define the $n \times s$-matrix $B_{v}$ by

$$
B_{v}(i, j)=\psi_{j}\left(\left[v, x_{i}\right]\right)
$$

Let $u_{1}, \ldots, u_{r}$ be a basis of $U$, and consider the field of rational functions $F=$ $k\left(U_{1}, \ldots, U_{r}\right)$. Let $u_{0}=U_{1} u_{1}+\cdots+U_{r} u_{r} \in F \otimes U$, and set $B_{U}=B_{u_{0}}$. (The entries of this matrix are linear polynomials in the $U_{k}$.)

Lemma 16. $U \cap G_{0} e \neq \emptyset$ if and only if the rank of $B_{U}$ is $s$.

Proof. Let $\mathfrak{z}_{0}(h)_{v}=\left\{x \in \mathfrak{z}_{0}(h) \mid[x, v]=0\right\}$. Let $x=\sum_{i} \alpha_{i} x_{i}$ and write $\alpha=$ $\left(\alpha_{1}, \ldots, \alpha_{n}\right)$. Then $x \in \mathfrak{z}_{0}(h)_{v}$ if and only if $\psi_{j}([v, x])=0$ for all $j$ if and only if $\alpha$. $B_{v}=0$. Hence $\operatorname{dim}\left[\mathfrak{z}_{0}(h), v\right]=\operatorname{rank}\left(B_{v}\right)$. So from what is said above, $U$ contains elements of $G_{0} e$ if and only if it contains elements $u$ such that $\operatorname{rank}\left(B_{u}\right)=s$, if and only if $\operatorname{rank}\left(B_{U}\right)=s$. 
Example 17. Here is an example of a matrix that has been produced by a calculation of this type:

$\begin{array}{cccccccccccccc}0 & U_{1} & 0 & 0 & 0 & 0 & 0 & 0 & 0 & -U_{6} & 0 & -U_{8} & 0 & 0 \\ 0 & 0 & U_{2} & 0 & 0 & 0 & U_{4} & 0 & -U_{5} & 0 & 0 & 0 & -U_{9} & 0 \\ 0 & 0 & 0 & 0 & 0 & U_{3} & 0 & 0 & 0 & -U_{7} & -U_{8} & 0 & 0 & 0 \\ 0 & 0 & U_{1} & 0 & 0 & 0 & 0 & -U_{4} & U_{6} & 0 & 0 & -U_{9} & 0 & 0 \\ 0 & 0 & 0 & U_{2} & 0 & 0 & -U_{3} & 0 & -U_{7} & 0 & U_{9} & 0 & 0 & 0 \\ 0 & 0 & 0 & U_{1} & 0 & 0 & 0 & U_{3} & U_{8} & U_{9} & 0 & 0 & 0 & 0 \\ U_{2} & 0 & 0 & 0 & 0 & 0 & 0 & 0 & 0 & 0 & -U_{5} & 0 & -U_{7} & 0 \\ 0 & 0 & 0 & 0 & 0 & 0 & 0 & 0 & 0 & 0 & 0 & 0 & 0 & -U_{8} \\ 0 & 0 & 0 & 0 & U_{4} & 0 & 0 & 0 & 0 & 0 & 0 & -U_{5} & -U_{6} & 0 \\ 0 & 0 & 0 & 0 & 0 & 0 & 0 & 0 & 0 & 0 & 0 & 0 & 0 & -U_{7} \\ 0 & 0 & 0 & 0 & 0 & 0 & 0 & 0 & 0 & 0 & 0 & 0 & 0 & U_{6} \\ 0 & 0 & 0 & 0 & 0 & 0 & 0 & 0 & 0 & 0 & 0 & 0 & 0 & U_{5} \\ 0 & 0 & 0 & 0 & 0 & 0 & 0 & 0 & 0 & U_{5} & U_{6} & U_{7} & U_{8} & U_{9} \\ 2 U_{1} & U_{2} & 0 & 0 & 0 & 0 & 0 & 0 & 0 & -U_{5} & 0 & -U_{7} & 0 & 0 \\ 0 & -U_{2} & 0 & 0 & U_{3} & U_{4} & 0 & 0 & 0 & -U_{5} & 0 & -U_{7} & 0 & 0 \\ -U_{1} & U_{2} & 0 & 0 & 0 & 0 & 0 & 0 & 0 & U_{5} & -U_{6} & U_{7} & -U_{8} & 0 \\ 0 & -U_{2} & 0 & 0 & 0 & -U_{4} & 0 & 0 & 0 & -U_{5} & 0 & 0 & U_{8} & -U_{9} \\ 0 & 0 & 0 & 0 & -U_{3} & U_{4} & 0 & 0 & 0 & U_{5} & U_{6} & -U_{7} & -U_{8} & 0\end{array}$.

It has rank 13 .

Lemma 16 reduces the problem of deciding whether $U \cap G_{0} e=\emptyset$ to computing the rank of a matrix over a function field, whose entries are linear polynomials. This is known as Edmonds' problem (see [23]). There is a straightforward algorithm, namely to do a Gaussian elimination over a function field. That method suffers from coefficient explosion, but works reasonably well if the matrices are not too big. For the matrices arising from the examples considered in [9] (including the adjoint representation of $E_{8}$, and a $\mathbb{Z} / 3 \mathbb{Z}$-grading of $E_{8}$ ), MAGMA was able to compute the rank.

Remark 18. The straightforward algorithm, based on Lemma 16, is not always used in the algorithm of [9]. In some cases a more efficient variant can be used; we will not go into that here. Furthermore, in some cases there are easy criteria that show that there is no inclusion (for example, if the orbits have equal dimension). Also, in [9], in order to loop over the orbit $W_{0} \cdot h$, a tree structure is used, due to Snow ([28]), where the nodes correspond to the elements of the orbit. A criterion is given that makes it possible, in many cases, to "prune" this tree, that is, if a space $U_{w}$, corresponding to a certain node of the tree, has no point of $G_{0} e^{\prime}$, and the criterion is fulfilled, then the same thing immediately follows for the entire subtree below, so that it can be skipped. This makes it possible to execute the algorithm also for cases where $W_{0}$ is very large, as for example, when considering the adjoint representation of $E_{8}$ (where $\left.\left|W_{0}\right|=696729600\right)$. 


\section{A Somewhat Larger Example}

Here we consider a $\theta$-group arising from the Lie algebra of type $E_{6}$. The Dynkin diagram is

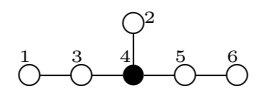

The corresponding Lie algebra $\mathfrak{g}$ is constructed exactly as in Example 12. The $\mathbb{Z}$-grading is also constructed in the same way: the simple root $\alpha_{4}$ has degree 1 , and the others have degree 0 . In this case $\mathfrak{g}_{0}$ is the direct sum of a semisimple Lie algebra of type $2 A_{2}+A_{1}$ and a 1 -dimensional torus. The space $\mathfrak{g}_{1}$ has dimension 18. Moreover, there are 17 orbits. Regarding the various methods described here to compute the orbit closures we remark:

- When computing the equations defining the closure of one particular orbit (Section 3), MAgma ran for 7 days and 8 hours, and got out of memory.

- Computing the degree of $G_{0}$ with Kazarnovskiı̌'s formula (as in Example 8) led to the number

$$
\frac{20 !}{72 \cdot(2 ! 2 !)^{2}} \frac{1}{169344000}=12471030 .
$$

If this number is correct, then the number of unknowns in the equations produced by the method of Section 4 is truly astronomical.

- The method of Section 5, which has been implemented in the SLA package of GAP4, computed all the orbit closures in 0.9 seconds.

This illustrates what in our view is a general pattern. Algorithms for computing with linear algebraic groups tend to be efficient if they use the correspondence with Lie algebras, and even more so if the combinatorics of roots and Weyl groups can be employed. On the other hand, algorithms that mostly rely on the geometric properties of the group tend to more generally applicable, but also to have difficulties when used in practice.

\section{References}

1. Dave Bayer and Mike Stillman. Computation of Hilbert functions. J. Symbolic Comput., 14(1):31-50, 1992.

2. W. Bosma, J. Cannon, and C. Playoust. The Magma algebra system. I. The user language. J. Symbolic Comput., 24(3-4):235-265, 1997. Computational algebra and number theory (London, 1993).

3. Dietrich Burde and Christine Steinhoff. Classification of orbit closures of 4dimensional complex Lie algebras. J. Algebra, 214(2):729-739, 1999.

4. C. Chevalley. Théorie des Groupes de Lie, Tome III. Théorèmes généraux sur les algèbres de Lie. Hermann, Paris, 1955.

5. D. Cox, J. Little, and D. O'Shea. Ideals, Varieties and Algorithms: An Introduction to Computational Algebraic Geometry and Commutative Algebra. Springer Verlag, New York, Heidelberg, Berlin, 1992. 
6. J. A. De Loera, B. Dutra, M. Köppe, S. Moreinis, G. Pinto, and J. Wu. Software for exact integration of polynomials over polyhedra. Comput. Geom., 46(3):232-252, 2013.

7. Harm Derksen and Gregor Kemper. Computational Invariant Theory. SpringerVerlag, Berlin Heidelberg New York, 2002.

8. The GAP Group. GAP - Groups, Algorithms, and Programming, Version 4.5, 2012. (http://www.gap-system.org).

9. W.A. de Graaf, E.B. Vinberg, and O.S. Yakimova. An effective method to compute closure ordering for nilpotent orbits of $\theta$-representations. J. Algebra, 371:38-62, 2012.

10. Willem A. de Graaf. Lie Algebras: Theory and Algorithms, volume 56 of NorthHolland Mathematical Library. Elsevier Science, 2000.

11. Willem A. de Graaf. Constructing algebraic groups from their Lie algebras. $J$. Symbolic Comput., 44:1223-1233, 2009.

12. Willem A. de Graaf. Computing representatives of nilpotent orbits of $\theta$-groups. $J$. Symbolic Comput., 46:438-458, 2011.

13. Willem A. de Graaf. SLA - computing with Simple Lie Algebras. a GAP package, 2013. (http://science.unitn.it/ degraaf/sla.html), version 0.13.

14. Joe Harris. Algebraic geometry, volume 133 of Graduate Texts in Mathematics. Springer-Verlag, New York, 1995. A first course, Corrected reprint of the 1992 original.

15. J. E. Humphreys. Linear Algebraic Groups. Springer Verlag, New York, Heidelberg, Berlin, 1975.

16. J. E. Humphreys. Reflection groups and Coxeter groups. Cambridge University Press, Cambridge, 1990.

17. Zbigniew Jelonek. On the effective Nullstellensatz. Invent. Math., 162(1):1-17, 2005.

18. V. G. Kac. Infinite Dimensional Lie Algebras. Cambridge University Press, Cambridge, third edition, 1990.

19. Noriaki Kawanaka. Orbits and stabilizers of nilpotent elements of a graded semisimple Lie algebra. J. Fac. Sci. Univ. Tokyo Sect. IA Math., 34(3):573-597, 1987.

20. B. Ya. Kazarnovskil. Newton polyhedra and Bezout's formula for matrix functions of finite-dimensional representations. Funktsional. Anal. i Prilozhen., 21(4):73-74, 1987. English translation: Functional Anal. Appl. 21 (1987), no. 4, 319321.

21. Witold Kraskiewicz and Jerzy Weyman. Geometry of orbit closures for the representations associated to gradings of Lie algebras of types $E_{6}, F_{4}$ and $G_{2}$. arXiv:1201.1102 [math.RT].

22. Witold Kraskiewicz and Jerzy Weyman. Geometry of orbit closures for the representations associated to gradings of Lie algebras of types $E_{7}$. arXiv:1301.0720 [math.RT].

23. L. Lovász. On determinants, matchings, and random algorithms. In Fundamentals of computation theory (Proc. Conf. Algebraic, Arith. and Categorical Methods in Comput. Theory, Berlin/Wendisch-Rietz, 1979), volume 2 of Math. Res., pages 565-574. Akademie-Verlag, Berlin, 1979.

24. G. D. Mostow. Fully reducible subgroups of algebraic groups. Amer. J. Math., 78:200-221, 1956.

25. V. L. Popov. The cone of Hilbert null forms. Tr. Mat. Inst. Steklova, 241(Teor. Chisel, Algebra i Algebr. Geom.):192-209, 2003. English translation in: Proc. Steklov Inst. Math. 241 (2003), no. 1, 177-194. 
26. V. L. Popov. Two orbits: when is one in the closure of the other? Tr. Mat. Inst. Steklova, 264(Mnogomernaya Algebraicheskaya Geometriya):152-164, 2009. English translation in: Proc. Steklov Inst. Math. 264 (2009), no. 1, 146-158.

27. Igor R. Shafarevich. Basic Algebraic Geometry 1. Springer-Verlag, Heidelberg, New York, 1994

28. D. M. Snow. Weyl group orbits. ACM Trans. Math. Software, 16(1):94-108, 1990.

29. Nicolas Spaltenstein. Classes unipotentes et sous-groupes de Borel, volume 946 of Lecture Notes in Mathematics. Springer-Verlag, Berlin, 1982.

30. Patrice Tauvel and Rupert W. T. Yu. Lie Algebras and Algebraic Groups. SpringerVerlag, Berlin Heidelberg New York, 2005.

31. È. B. Vinberg. The classification of nilpotent elements of graded Lie algebras. Dokl. Akad. Nauk SSSR, 225(4):745-748, 1975.

32. È. B. Vinberg. The Weyl group of a graded Lie algebra. Izv. Akad. Nauk SSSR Ser. Mat., 40(3):488-526, 1976. English translation: Math. USSR-Izv. 10, 463-495 (1976).

33. E. B. Vinberg. Classification of homogeneous nilpotent elements of a semisimple graded Lie algebra. Trudy Sem. Vektor. Tenzor. Anal., (19):155-177, 1979. English translation: Selecta Math. Sov. 6, 15-35 (1987).

34. È. B. Vinberg and V. L. Popov. Invariant theory. In Algebraic geometry, 4 (Russian), Itogi Nauki i Tekhniki, pages 137-314. Akad. Nauk SSSR Vsesoyuz. Inst. Nauchn. i Tekhn. Inform., Moscow, 1989. English translation in: V. L. Popov and È. B. Vinberg, Invariant Theory, in: Algebraic Geometry IV, Encyclopedia of Mathematical Sciences, Vol. 55, Springer-Verlag, Berlin, 1994, pp. 123-284. 